SAND98-1106

Unlimited Release

Printed June 1998

\title{
Physico-Chemical Stability of Solid Surfaces: Final Report
}

RECEIVED

JUN 26998

OSTI

Terry A. Michalske, Dwight R. Jennison, Peter J. Feibelman, Jack E. Houston, Gary L. Kellogg

Prepared by

Sandia National Laboratories

Albuquerque, New Mexico 87185 and Livermore, California 94550

Sandia is a multiprogram laboratory operated by Sandia Corporation,

a Lockheed Martin Company, for the United States Department of

Energy under Contract DE-AC04-94AL85000.

Approved for public release; further dissemination unlimited.

\section{iin Sandia National Laboratories}

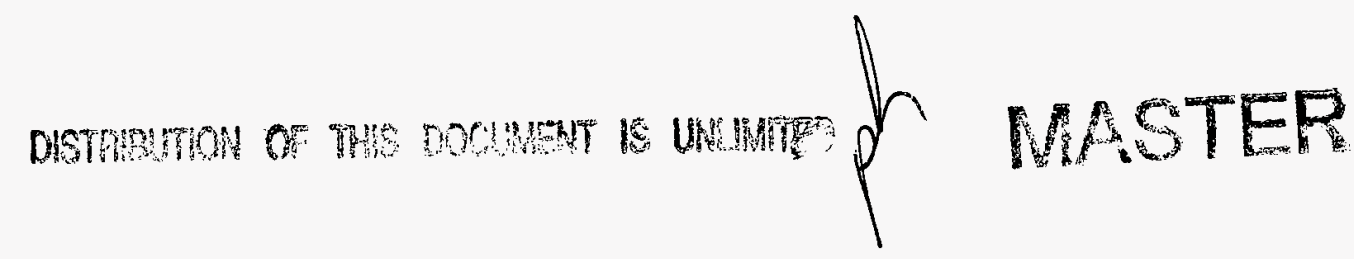


Issued by Sandia National Laboratories, operated for the United States Department of Energy by Sandia Corporation.

NOTICE: This report was prepared as an account of work sponsored by an agency of the United States Government. Neither the United States Government nor any agency thereof, nor any of their employees, nor any of their contractors, subcontractors, or their employees, makes any warranty, express or implied, or assumes any legal liability or responsibility for the accuracy, completeness, or usefulness of any information, apparatus, product, or process disclosed, or represents that its use would not infringe privately owned rights. Reference herein to any specific commercial product, process, or service by trade name, trademark, manufacturer, or otherwise, does not necessarily constitute or imply its endorsement, recommendation, or favoring by the United States Government, any agency thereof, or any of their contractors or subcontractors. The views and opinions expressed herein do not necessarily state or reflect those of the United States Government, any agency thereof, or any of their contractors.

Printed in the United States of America. This report has been reproduced directly from the best available copy.

Available to DOE and DOE contractors from

Office of Scientific and Technical Information

P.O. Box 62

Oak Ridge, TN 37831

Prices available from (615) 576-8401, FTS 626-8401

Available to the public from

National Technical Information Service

U.S. Department of Commerce

5285 Port Royal Rd

Springfield, VA 22161

NTIS price codes

Printed copy: A03

Microfiche copy: A01

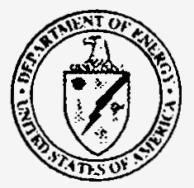


SAND98-1106

Unlimited Release

Printed June 1998

\title{
Physico-Chemical Stability of Solid Surfaces: Final Report
}

\author{
Terry A. Michalske, Dwight R. Jennison, Peter J. Feibelman, \\ Jack E. Houston, Gary L. Kellogg \\ Surface and Interface Sciences Department \\ Sandia National Laboratories \\ P.O. Box 5800 \\ Albuquerque, NM 87185-1413
}

\begin{abstract}
The application of physico-chemical phenomena to either increase machinability of hard materials, improve the wear resistance of cutting surfaces, or enhance sintering of particle compacts can have large economic impact on technologies ranging from materials forming processes to oil well drilling. Unfortunately, the broad application of these physico-chemical principles is limited by our ability to predict the optimum conditions for a wide variety of materials surfaces. Predictive models must be built upon understanding of the elementary events involved in surface damage and mobility. We have developed a new approach to examine the fundamental mechanisms controlling physico-chemical surface stability that combines: 1) atomic-scale control of surface contact forces and displacements under well controlled adsorbate conditions using the Interfacial Force Microscope, 2) atomic-level imaging of surface and nearsurface structure and defects using Field Ion Microscopy, and 3) first-principles modeling of the effect of surface stress on adsorbate bonding interactions and the subsequent generation of surface damage. This unique combination of approaches has provided new insights into observed physico-chemical phenomena and provided the basis for developing true predictive models that are needed for wide application of these important new approaches to modifying the surface sensitive properties of materials.
\end{abstract}




\section{Introduction}

The presence of a chemically active environment is known to have a profound effect on the mechanical strength, fatigue, and wear resistance of metal and ceramic materials. For example, the embrittlement of metals by hydrogen or liquid metal adsorbates has been responsible for many costly structural disasters. Similarly, simple aqueous environments drastically reduce the structural integrity of ceramic components and prohibit their application in many load-bearing applications. Although the combined chemical/mechanical (physico-chemical) effects on materials are often associated with undesirable outcomes, other work has demonstrated that these effects can also be used to benefit a wide range of materials processes and applications. Examples of the technological application of surface-mediated materials processes include comminution, grinding, machining and drilling. Liquid metals, which are known to embrittle steels and titanium alloys, have been used to facilitate the cutting of these hard materials. It has also been demonstrated that the wear of drill bit materials for rock boring can be greatly reduced by choosing the proper aqueous chemistry. Recent studies indicate that physico-chemical principles may even be applied to improve the sintering of particle compacts.

These examples would suggest that a broad range of technologically important materials processes could be improved by applying physico-chemical principles. Given these dramatic examples one must ask: Why have physico-chemical principles not been applied for the wide benefit of technologically important manufacturing processes? The answer is that we lack mechanistic-based models to predict the proper conditions needed to achieve the desired result for a wide range of materials. To date, the demonstrations of physico-chemical principles have been made using rather complex processes (drilling, grinding, sintering). The observations derived from these complex systems have not lent themselves to the identification of elementary processes that are at the root of these surfacemediated phenomena. Wide application of physico-chemical principles will require the 
development of predictive models the are built from fundamental understanding. These fundamental understandings can only be achieved by combining atomic-level experimental methods with accurate first-principles computational studies.

\section{Technical Issues}

Simply stated, the important issues that must be resolved in order to model physicochemical phenomena concern the effect of surface adsorbates on the deformation characteristics and surface mobility of solids. In turn we must also develop models that provide understanding of the roll of mechanical stress on adsorption processes. These are very broad issues; however, they can be addressed by seeking answers to several specific questions.

* How does surface strain act to promote adsorption?

* What is the role of surface-active species in controlling the formation and mobility of dislocations in metals?

* What role do defects (vacancies, crystal terrace steps etc.) play in adsorption and deformation processes?

* How can the adsorbate bond weaken (or strengthen) the interatomic bonds in the substrate surface or near surface?

* How can the formation of chemical bonds to the surface affect the mobility of surface atoms?

The answers to these and other important questions are needed to form the basis for predictive models for physico-chemical processes. In this work, we address these atomiclevel processes by developing a new approach that combines atomic-level measurement and modeling.

\section{Technical Approach}

We employed a new approach to examine the fundamental mechanisms controlling physico-chemical surface stability that combines: 1) atomic-scale control of surface contact 
forces and displacements under well controlled adsorbate conditions using the Interfacial Force Microscope, 2) atomic-level imaging of surface and near-surface structure and defects using Field Ion Microscopy, and 3) first-principles modeling of the effect of surface stress on adsorbate bonding interactions and the subsequent generation of surface damage. This unique combination of approaches provided new insights into observed physico-chemical phenomena and provided the basis for developing true predictive models that are needed for wide application of these important new approaches to modifying the surface sensitive properties of materials.

The Interfacial Force Microscope (IFM), which was developed at Sandia, utilizes a unique force-balance feed back sensor that is mechanically stable over the entire range of forces from attractive to repulsive contact. Using the IFM in a controlled environment chamber, a well characterized probe tip can be brought into contact with a well characterized substrate surface to produce controlled and measurable surface and near-surface forces and deformations with nanonewton force control and angstrom displacement resolution. In this way, the onset of plasticity can be determined directly as a function of surface adsorbate conditions, temperature, or electrical bias. Substrate deformation will be imaged on an atomic-scale by operating the IFM in the scanning mode. This direct, atomic-scale measure of the surface deformation characteristics can be conducted for a wide range of materials combinations and environmental conditions including liquid or electrochemical conditions.

These direct atomic-scale measurements can be correlated with results of accurate first-principles calculations to examine adsorbate binding interactions in the presence of applied mechanical stress. Massively-parallel (MP) computing methods developed at Sandia have recently yielded unprecedented advances in LDF problem size capability. For the first time it will be possible to study unit cells of sufficient size, of the order of 100 atoms, so that adsorbate interactions can be studied on extended surfaces. We use these new capabilities to examine the elementary processes in the combined chemical/mechanical interaction of 
adsorbates on solid surfaces by studying the interdependence of mechanical stress and surface reactivity.

We have chosen to examine in parallel two classes of surface active adsorbates: gas phase molecular species and adsorbed metal overlayers. These two systems will allow us to address important technological problems such as hydrogen and ammonia induced stress corrosion cracking of metals and liquid metal embrittlement.

\section{Stress Enhanced Surface Chemistry}

In this portion of the project we directly studied the role of mechanical stress on adsorption of reactive, gas phase molecules. The calculations that we conducted both uniformly stresses surfaces and surfaces where the mechanical stress was induced by the existence of a crystal dislocation. In each case we examined the $\mathrm{Pt}(111)$ surface. Three types of dislocations were studied: threading, misfit, and bright star. Atom locations determined by EAM (John Hamilton, 8718) were used to produce clusters of approx. 100 atoms. We examined adsorbate binding $\left(\mathrm{NH}_{3}\right.$ and $\left.\mathrm{CO}\right)$ near dislocation cores and compared it to adsorption on extended surfaces. Significant differences in adsorbate binding, over $0.1 \mathrm{eV}$, were found to occur near the dislocation cores. We are currently conducting calculations to determine the origin of the increased binding to dislocation core structures. In one set of these calculations, we separately explored the role of mechancial strain on the binding energy for these adsorbates. Interestingly, ammonia binds more strongly when the slab is compressed or stretched by $1 \%$, while $\mathrm{CO}$ loses bonding upon compression and gains binding upon tensile strain. We are continuing these studies to examine the effect of changing from bridge to hcp sites near a dislocation core.

\section{Adsorbate Enhanced Surface Diffusion}

It is well known that the sintering of metals and ceramics can be strongly affected by the composition of the ambient environment. We conducted the first quantum chemical 
calculations to show how adsorbates can enhance surface self-diffusion in metals (1). We found that an adsorbed $\mathrm{H}$ decreases the self-diffusion barrier by a factor of three. The physical explanation for this involves the change in bond order - bond length as a function of coordination number. When the hydrogen bonds to the diffusing adatom, it weakens and lengthens its bonds to the substrate. In this way the adsorbate acts like a "sky hook" pulling the adatom further from the surface where it feels a smaller electronic corrugation due to the periodic structure of the substrate. This lower electronic corrugation reduces the diffusional barrier. We also conducted experimental studies using Field Ion Microscopy (FIM) to directly observe the effect of adsorbed hydrogen on self-diffusion of $\mathrm{Pt}(2,3)$. These first ever measurements show that hydrogen strongly accelerated diffusion. The observed first order coverage dependence is consistent with the chemical mechanism predicted by our previous first-principles calculations.

\section{Liquid Metal Embrittlement}

We formulated a theoretical approach to modeling the mechanisms responsible for embrittlement by liquid metals. We put forward a new mechanistic model that can be used to predict which metals can act a embrittling agents (4). This new approach begins by addressing a well-known and technologically important embrittlement couple - $\mathrm{Al} / \mathrm{Ga}$. (Mercury has the same effect and is therefore forbidden from aircraft with aluminum air frames). It has be supposed that $\mathrm{Ga}$ diffuses to the crack tip to affect the $\mathrm{Al}$ bonds but the question is, "Why doesn't the Ga simply bond across the crack tip and stop cracking." Our new theory suggests that the high valance state promotion energy for Ga prevents it from forming many bonds and therefore precludes the formation of a bridge across the crack tip. This represents the first model to address liquid metal embrittlement from a chemical view and provides a new approach to predict embrittlement effects. We then conducted a series of first-principles calculations to verify this new model. Our results prove that $\mathrm{Ga}$ wets the low Miller index Al planes. Consistent with a thermodynamic ("Griffith criterion") basis for the observation that Ga causes single-crystal Al to crack along (100) planes, the Ga-covered 
(100) plane has the lowest surface energy. This represents a compromise between weaker Ga binding on closerpacked $\mathrm{Al}$ surfaces and higher clean surface surface-energies on moreopen ones. The second adsorbed $\mathrm{Ga}$ layer, on $\mathrm{Al}(100)$, lies rather highabove the first (compared to the distance of the first layer from the Al's) and is weakly bound to it. This result is consistent with the idea that $\mathrm{Ga}$ is not suited for welding one side of an incipient crack to the other. It also means that the barrier to diffusion of secondlayer $\mathrm{Ga}$ adatoms is quite low. We estimate it to be on the order of $10 \mathrm{meV}$. This is consistent with rapid diffusion of Ga's to a cracktip, a necessity if $\mathrm{Ga}$ is consumed by adsorption on freshly exposed $\mathrm{Al}$ as the crack propagates.

After making the necessary physical and electronic modifications to the Interfacial Force Microscope that permit operation in Ultra High Vacuum (UHV), we designed and conducted a series of experiments to explore the role of liquid metal adsorbes on the mechanical properties of clean metal surfaces and interfaces. In these experiments, we were able, for the first time, to clean the IFM probe tip (W) and the substate surface (Au) and directly measure the mechanical interactions as these metal surfaces were brought together in UHV conditions. In conventional force microscopy, the proximity of the tip is determined by physically contacting the substrate prior to any actual force measurements. Since clean metal surfaces interact so strongly, we had to invent a new method by which data could be obtained on the first approach to the substate. By applying a bias voltage to the tip, we were able to guide it into position for the first measurement using the field emitted electron intensity. With this new experimental technique developed, we conducted experiments that contrast the mechanical interaction for a clean Au surface against one that has be covered with monolayers of $\mathrm{Hg}$. Since $\mathrm{Hg}$ is a classic embrittling agent for gold, we used these experiments to study the details of the embrittling process. Our results showed, contrary to expectations, that monolayer coverage of $\mathrm{Hg}$ actually strengthened the mechanical bond between the clean metals by greater than a factor of six. In addition, these measurements showed for the first time that the liquid metal actually softens the Au substate surface, allowing an adhesive 
instability as the outermost atomic layer of metal atoms "jump" across to form the adhesive bond.

These atomic-level mechanical measurements were complemented by macroscopic wear studies conducted by our university collaborator, Prof. Shchukin. Using a unique microscrotching apparatus, he demonstrated the effect of liquid embrittling metals on the surface wear of $\mathrm{Al}(5)$. His results show a dramatic softening of the $\mathrm{Al}$ surface by $\mathrm{Ga}$ (a known embrittling agent for $\mathrm{Al}$ ). This softening is consistent with our atomic-scale IFM measurements for the $\mathrm{Hg} / \mathrm{Au}$ system.

\section{Summary}

This study used atomic-scale experimental and computational techniques to address the origins of physico-chemical phenomena. We demonstrated that this approach could provide new insight into long-standing materials science problems. In many cases, the results go counter to conventional wisdom. In all cases, the new fundamental insight provides a solid foundation on which to construct predictive materials models.

\section{References}

1) R. Stumpf and P. Feibelman, Phys. Rev. B Vol. 51, 13748 (1995)

2) G. L. Kellogg, Phys. Rev. B Vol. 55, 7206 (1997)

3) G. L. Kellogg, Phys. Rev. Let. Vol. 79, 4417 (1997)

4) R. Stumpf and P. Feibelman, Phys. Rev. B Vol. 54, 5145 (1996)

5) E. Shchukin, T. Michalske, R. Green and I Vidensky, J. Mat. Res. (in press) 


\section{DISTRIBUTION:}

3 MS1413 T. A. Michalske, 1114

1 MS1413 D. R. Jennison, 1114

1 MS1413 P. J. Feibelman, 1114

1 MS1413 J. E. Houston, 1114

1 MS1413 G. L. Kellogg, 1114

2 MS0188 LDRD Office, 4523

1 MS9018 Central Technical Files, 8940-2

2 MS0899 Technical Library, 4916

2 MS0619 Review \& Approval Desk, 12690

for DOE/OSTI 


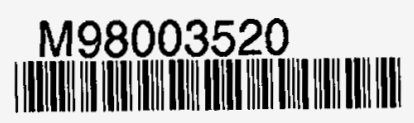

Report Number (14) SAND $-98-1 / 106$
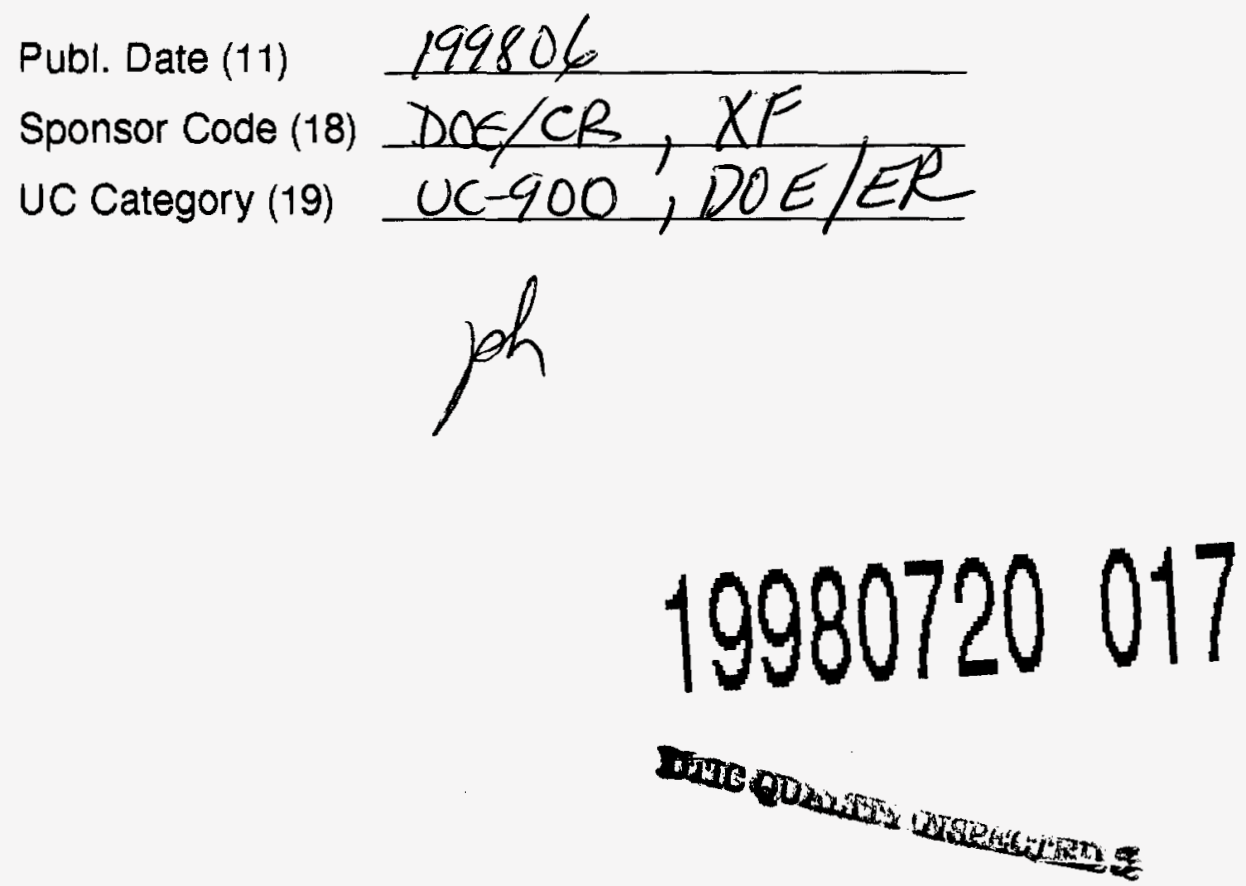